\title{
AN INTEGRATED MODEL FOR ANAPHORA RESOLUTION
}

\author{
Ruslan Mitkov \\ Institute of Mathematics \\ Acad. G. Bonchev str. bl.8, 1113 Sofia, Bulgaria
}

\begin{abstract}
The paper discusses a new knowledgebased and sublanguage-oriented model for anaphora resolution, which integrates syntactic, semantic, discourse, domain and heuristical knowledge for the sublanguage of computer science. Special attention is paid to a new approach for tracking the center throughout a discourse scgment, which plays an important role in proposing the most likcly antecedent to the anaphor in case of ambiguity.
\end{abstract}

\section{INTRODUCTION}

Anaphora resolution is a complicated problem in computational linguistics. Considerable rescarch has bcen done by computational linguists ([Carboncll \& Brown 88], [Dahl \& Ball 90], [Frederking \& Gchrke 87], [Hayes 81], [Hobbs 78], [Ingria \& Stallard 89], [Preuß et al. 94], [Rich \& LuperFoy 88], [Robert 89]), but no complete theory has cmerged which offers a resolution proccdure with success guarantecd. All approaches developed - even if we restrict our attention to pronominal anaphora, which we will do throughout this paper from purely syntactic ones to highly semantic and pragmatic ones, only provide a partial treatment of the problem.

Given the complexity of the problem, we think that to secure a comparatively successful handling of anaphora resolution one should adhere to the following principles: 1) restriction to a domain (sublanguage) rather than focus on a particular natural language as a whole; 2) maximal use of linguistic information integrating it into a uniform architecture by means of partial theorics. Some more recent treatments of anaphora ([Carbonell \& Brown 88], [PreuB et al. 94], [Rich \& LuperFoy 881) do express the idea of "multi-level approach", or "distributed architecturc", but their ideas a) do not secm to capture enough discourse and heuristical knowledge and b) do not concentrate on and investigate a concrete domain, and thus risk being too general. We have tried nevertheless to incorporate some of their ideas into our proposal.

\section{THE ANAPHORA RESOLUTION MODEL}

Our anaphora resolution model integrates modules containing different types of knowledge - syntactic, scmantic, domain, discourse and heuristical knowledge. All the modules share a common representation of the current discourse.

The syntactic module, for example, knows that the anaphor and antecedent must agree in number, gender and person. It checks if the c-command constraints hold and cstablishes disjoint reference. In cases of syntactic parallelism, it prefers the noun phrase with the same syntactic role as the anaphor, as the most probable antecedent. It knows when cataphora is possible and can indicate syntactically topicalized noun phrases, which are more likely to be antecedents than non-topicalized ones.

The semantic module checks for semantic consistency between the anaphor and the possible anteccdent. It filters out semantically incompatible candidates following the current verb semantics or the animacy of the candidate. In cases of scmantic parallelism, it prefers the noun phrase, having the same semantic role as the anaphor, as a most likely antecedent. Finally, it generates a set of possible antecedents whenever nccessary.

The domain knowledge module is practically a knowledge base of the concepts of the domain considered and 
the discourse knowledge module knows how to track the center throughout the current discourse segment.

The heuristical knowledge module can sometimes be helpful in assigning the antecedent. It has a set of useful rules (c.g. the antecedent is to be located preferably in the current sentence or in the previous one) and can forestall certain impractical scarch procedures.

The use of common sense and world knowledge is in general commendable, but it requires a huge knowledge base and sel of inference rules. The present version of the model does not have this module implemented; its development, however, is cnvisaged for later stages of the project.

The syntactic and semantic modules usually lilter the possible candidates and do not propose an antecedent (with the cxception of syntactic and scmantic parallelism). Usually the proposal for an antecedent comes from the domain, heuristical, and discourse modules. The latter plays an important role in tracking the center and proposes it in many cases as the most probable candidate for an antecedent.

Figure I illustrates the general structure of our anaphora resolution model.

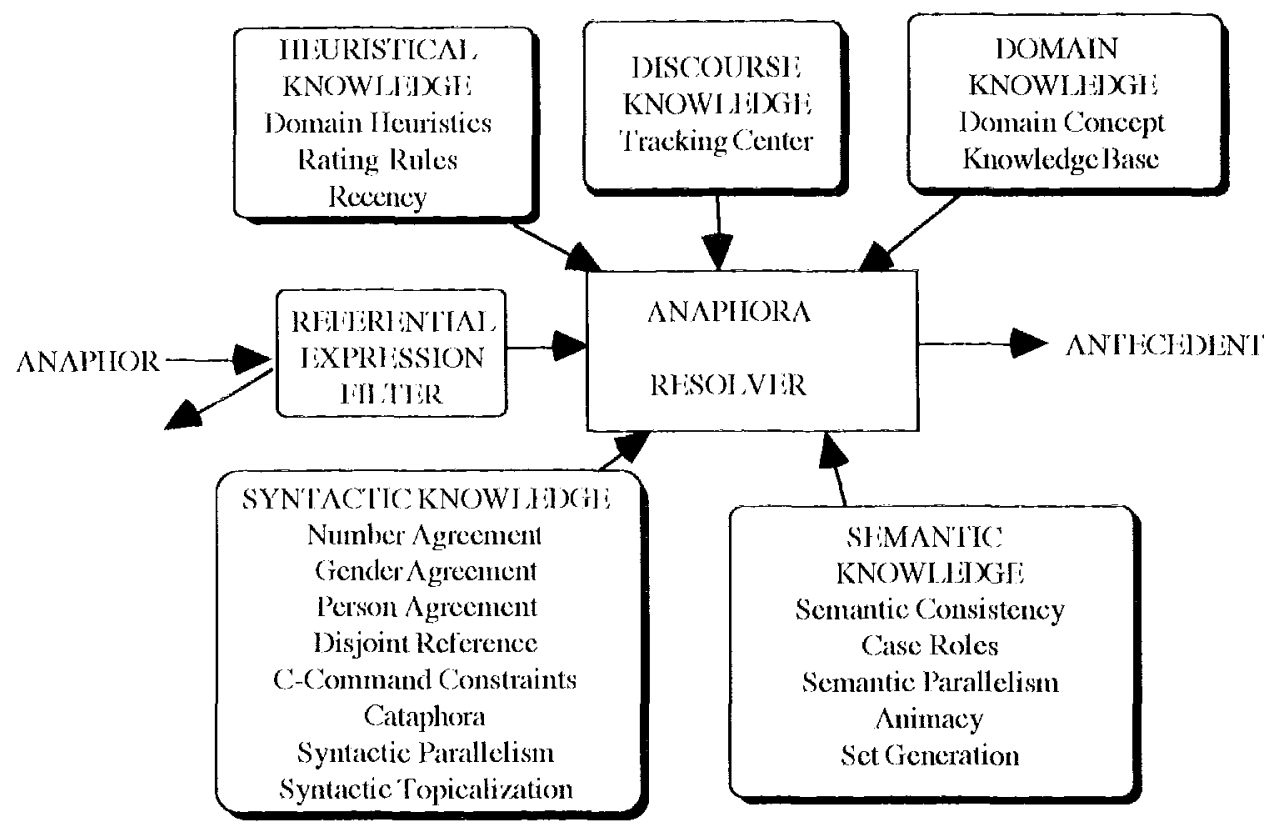

Figure 1: Anaphora resolution model

\section{THE NEED FOR DISCOURSE CRITERIA}

Although the syntactic and semantic criteria for the selection of an antecedent are already very strong, they are not always sufficient to discriminatc among a set of possible candidates. Moreover, they scrve more as filters to climinate unsuitable candidates than as proposcrs of the most likely candidate. $\Lambda$ dditional critcria are therefore needed.

As an illustration, consider the following text.

\footnotetext{
Chapter 3 discusses these additional or auxiliary storage deviecs, which are similar to our own domestic tape eassettes and record dises. Figure 2 illustrates their connection to the main central memory.
} 
In this discourse segment neither the syntactic, nor the scmantic constraints can eliminate the ambiguity between "storage devices", "tapc casscttes" or "record discs" as antecedents for "their", and thus cannot turn up a plausible antecedent from among these candidates. A human reader would be in a better position since he would be able to identify the central concept, which is a primary candidate for pronominalization. Correct identification of the antecedent is possible on the basis of the pronominal reference hypothesis: in cvery sentence which contains one or more pronouns must have one of its pronouns refer to the center ${ }^{1}$ of the previous sentence. Therefore, whenever we have to find a referent of a pronoun which is alone in the sentence, we have to look for the centered element in the previous sentence.

Following this hypothesis, and recognizing "storage devices" as the center, an anaphora resolution model would not have problems in picking up the center of the previous sentence ("storage devices") as antecedent for "their".

We see now that the main problem which arises is the tracking of the center throughout the discourse segment. Certain ideas and algorithms for tracking focus or center (c.g. [Brennan et al.871) have been proposed, provided that one knows the focus or center of the first sentence in the segment. Howcver, they do not try to identify this center. Our approach determines the most probable center of the first sentence, and then tracks it all the way through the segment, correcting the proposed algorithm at each step.

TRACKING THE CENTER IN THE SUBLANGUAGE OF COMPUTER SCIENCE

Identifying ecnter can be very helpful in

\footnotetext{
1 Though "center" is an utterance-specific notion, we refer to "sentence center", because in many cases the centers of the utterances a sentence inay consist of, coincide. In a complex sentence, however, we distinguish also "clause centers"
}

anaphora resolution. Usually a center is the most likely candidate for pronominalization.

There are different views in literature regarding the preferred candidate for a center (focus). Sidner's algorithm ([Sidner 81]), which is based on thematic roles, prefers the theme of the previous sentence as the focus of the current sentence. This view, in general, is advocated also in ([Allen87]). PUNDIT, in its current implementation, considers the entire previous utterance to be the potential focus ([Dahl\&Ball 90]). Finally, in the centcring literature ([Brennan et al. 871), the subject is generally considered to be preferred. We have found, however, that there are many additional interrclated factors which influence upon the location of the center.

We studied the "behaviour" of center in various computer scicnce texts $(30$ different sources totally exceeding 1000 pages) and the empirical observations cnabled us to develop efficient sublanguage-dependent heuristics for tracking the center in the sublanguage of computer science. We summarize the most important conclusions as follows:

1) Consider the primary candidates for center from the priority list: subject, object, verb phrasc.

2) Prefer the NP, representing a domain concept to the NPs, which arc not domain concepts.

3) If the verb is a member of the Verb_set $=$ \{discuss, present, illustrate, summarize, cxamine, describe, definc, show, check, develop, review, report, outline, consider, investigate, explore, assess, analyzc, synthesize, study, survey, deal, cover $\}$, then consider the object as a most probable center.

4) If a verbal adjective is a member of the Adj_set $=$ \{defined, called, so-called $\}$, then consider the NP they refer to as the probable center of the subsequent clausc/current sentence. 
5) If the subject is "chapter", "scction", "table", or a personal pronoun - "I", "we", "you", then consider the object as most likely center.

6) If a NP is repcated throughout the discourse section, then consider it as the most probable center.

7) If an NP occurs in the head of the section, part of which is the current discourse segment, then consider it as the probable center.

8) If a NP is topicalized, then consider it as a probable center.

9) Prefer definite NPs to indefinite oncs.

10) Prefer the NPs in the main clause to NPs in the subordinate clauses.

11) If the sentence is complex, then prefer for an antecedent a noun phrase from the previous clause within the same sentence.

As far as rule 1 is concened, we found that the subject is a primary candidate for center in about $73 \%$ of the cases. The second most likely center would be the object $(25 \%)$ and the third most likely one the verb phrase as a whole $(2 \%)$. Therelore, the priority list [subject, object, verb phrase] is considered in tcrms of the apriori cstimated probability.

There are certain 'symptoms' which determine the subject or the object as a center with very high probability. Cases in point are 3) and 5). Other cases are not so certain, but to some extent quite likely. For example, if a non-concept NP is in subject position and if a repeated concept $\mathrm{NP}$, which is also in a head, is in object position, it is almost certain that the latter is the unambiguous center. Morcover, certain preferences are stronger than others. For example an NP in subject position is preferred over an NP in a section head, but not in subject position.

We have made use of our empirical results (with approximating probability measures) and AI techniques to devclop a proposer module which identifies the most likely center. We must point out that even if we do not need one for immediate antecedent disambiguation, a center must still be proposed for each sentence. Or else we will have to go all the way back to track it from the beginning of the segment when one is needed later on.

The rules 1)- 11) should be ordered according to their priority - a problem, which is being currently investigated.

Tracking the center in a discourse segment is very important since knowing the center of each eurrent sentence helps in many cases to make correct decisions about an antecedent in the event that syntactic and semantic constraints cannot discriminate among the available candidates.

AN ARTIFICIAL IN'TELLIGENCE
APPROACH FOR CALCULATING
THE PROBABILITY OF A NOUN
(VERB) PHRASE TO BE IN THE
CENTER

On the basis of the results described in the previous section, we use an artificial intelligence approach to determine the probability of a noun (verb) phrase to be the center of a sentence. Note that this approach allows us to calculate this probability in every discourse sentence, including the first one and to propose the most probable center. This approach, combined with the algorithm for tracking the center ([Brennan et al. 87|), is expected to yickd improved results.

Our model incorporates an AI algorithm for calculating the probability of a noun (verb) phrase to be in the center of a discourse segment. The algorithm uses an inference engine based on Bayes' theorem:

$$
P\left(I_{K} \mid A\right)=\frac{P\left(H_{K}\right) P\left(A \mid H_{K}\right)}{P\left(H_{I}\right) P\left(A \mid H_{Y}\right)}
$$

$$
\text { for } \mathrm{K}=1,2, \ldots
$$

Under the conditions of our model Bayes' theorem allows the following 
interpretation: there are only two possible hypotheses for a certain noun (verb) phrase - that it is the center of the current sentence (clause) or that it is not. Let $\mathrm{H}_{Y}$ be the positive, while $\mathrm{H}_{\mathrm{N}}$ - the negative hypothesis. If we call the presence of some of the pieces of evidence, described in the previous section, a "symptom", then let A denote the occurrence of that symptom with the examined phrase. $\mathrm{P}\left(\mathrm{AlH} \mathrm{H}_{\mathrm{Y}}\right)$ would be the apriori probability of the symptom $A$ being observed with a noun (verb) phrase which is the center (we will henceforth refer to this factor as $\mathrm{PY})$. By analogy $\mathrm{P}\left(\mathrm{A} / \mathrm{H}_{\mathrm{N}}\right)$ is the probability of the symptom being observed with a phrase which is not the center (henceforth referred to as $\mathrm{P}_{\mathrm{N}}$ ). The aposteriori probability $\mathrm{P}\left(\mathrm{H}_{\mathrm{K}} \mid \mathrm{A}\right)$ is defined in the light of the new piece of evidence the presence of an empirically obtained symptom, indicating the higher probability the examined phrase to be in the center of the discourse segment.

In other words, inference engine bascd on Bayes' theorem draws an inference in the light of some new piece of evidence. This formula calculates the new probability, given the old probability plus some new piece of evidence.

Consider the following situation. According to our investigation so far, the probability of the subject being a center is $73 \%$. Additional evidence (symptom), e.g. if the subject represents a domain concept, will increase the initial probability. If this NP is also the head of the section, the probability is increased further. If the NP occurs more than once in the discourse segment, the probability gets even higher.

An estimation of the probability of a subject, (direct or indirect) object or verb phrase (the only possible centers in our texts) to be conters, can be represented as a predicate with arguments:

center $\left(X, P_{1}\right.$, [symptom (weight factor $_{1}$, weight factor $\left.{ }_{2}\right), \ldots$, symptom ${ }_{\mathrm{N}}$ (weight factor $\mathrm{N}_{1}$, weight factor $\mathrm{N}_{2}$ )] )

where center ( $X, I$, list) represents the estimated probability of $X$ to be the center of a sentence (clause), $X \in$ \{subject, object $_{1}$, object $_{2}, \ldots$ verb phrase $\}$ and $P_{1}$ is the initial probability of $X$ to be the center of the sentence (clause).

Weight factor ${ }_{1}$ is the probability of the symptom being observed with a noun (verb) phrase which is the center $\left(\mathrm{P}_{\mathrm{Y}}\right)$. Weight factor $_{2}$ is the probability of the symptom being observed with a noun (verb) phrase wnich is not the center $\left(\mathrm{P}_{\mathrm{N}}\right)$.

Following our preliminary results, we can write in Prolog notation:

center (object, 25, [symptom (verb_set, 40, 3), symptom (subject_sct, 40,2), symptom (domain_concept $(95,80)$, symptom (repeated, 10, 5), symptom (headline, 10, 9)1, symptom (topicalized, $6,2)$, symptom (main_clause $(85,30)$, symptom (definite_form $(90,70)]$ )

center (subject, 73, [symptom (domain concept $(95,70)$, symptom (repeated, 10, 4), symptom (hcadlinc, 10,8 ), symptom (topicalized, 10,3), symptom (main_clause $(85,30)$, symptom (definite_form $(85,20)]$ ).

The first fact means that the object is the center in approximately $25 \%$ of the cascs. Moreover, it suggests that in $40 \%$ of the cases where the center is the object, the verb belongs to the set of verbs \{discuss, illustrate, summarizc, cxamine, describe, define... $\}$ and it is possible with $3 \%$ probability for the verb to be a member of this set while the center of the sentence is not the object.

The above Prolog facts are part of a sublanguage knowledge base.

The process of estimating the probability of a given phrase being the center of a sentence (clause), is repetitive, beginning with an initial cstimate and gradually working lowards a more accurate answer. More systematically, the "diagnostic" process is as follows:

- start with the initial probability

- consider the symptoms one at a time

- for each symptom, update the current probability, taking into account: a) whether the sentence has the symptom and b) the weight factors $\mathrm{PY}_{\mathrm{Y}}$ and $\mathrm{P}_{\mathrm{N}}$. 
The probability for an NP to be the center is calculated by the inference engine represented as a Prolog program (left out here for reasons of space), which operates on the basis of the sublanguage knowledge base and the "local" knowledge base. The latter gives information on the current discourse segment. Initially, our program works with manual inpuls. The local knowledge base can be represented as Prolog lacts in the following way:

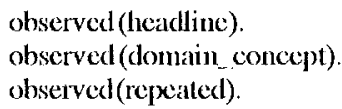

'The inference engine's task is to match the expected symptoms of the possible syntactic function as center in the knowledge batse of the sentence's actual symptoms, and produce a list of (rcasonably) possible candidates.

THE PROCEDURF:

AN INTEGRATED KNOWLEDGE
$\underline{\text { APPROACH }}$

Our algorithm for assigning (proposing) an antecedent to an anaphor is sublanguage-oriented because it is based on rules resulting from studies of computer science texts. It is also knowledge-based because it uses at least syntactic, semantic and discourse knowledge. Discourse knowledge and especially knowing how to track the center play a decisive role in proposing the most likely antecedent.

The initial version of our project handles only pronominal anaphors. However, not all pronouns may have specific reference (as in constructions like "it is necessary", "it should be pointed out", "it is clcar", ...). So before the input is given to the anaphor resolver, the pronoun is checked to ensure that it is not a part of such grammatical construction. This function is carricd out by the "referential expression filter".

The procedure for proposing an antecedent to an anaphot operates on discourse segments and can be described informally in the following way:
1) Propose the center of the first sentence of the discourse segment using the method described.

2) Use the algorithm proposed in [Brennan ct al. 87], improved by an additional estimation of the correct probability supplied by our method, in order to track the center throughout the discourse segment (in case the anaphor is in a complex sentence, identify clause centers too).

3) Usc syntactic and semantic constraints to climinate antecedent candidates.

4) Propose the noun phrase that has been filtered out as the antecedent in case no other candidates have come up; otherwise propose the center of the preceding sentence (clause) as the antecedent .

The information oblatined in 1) and 2) may not be used; however, it may be vital for proposing an antecedent in case of ambiguily.

To illustrate how the algorithm works, consider the following sample text:

\section{SYSIIMM PROCIRAMS}

We should note that, unlike user programs, system programs such as the supervisor and the language translator should not have to be translated every lime they are used, otherwise this would result in a serious increase in the time spent in processing a user's program. System prograns are usually written in the asscmbly version of the machine language and are translated once into the machine code itself. lirom then on they can be loaded into inemory in machine code without the need for any immediate transtation phases. They are written by specialist programmers, who are callod system programmers and who know a great deal about the computer and the computer system for which their programs are written. They know the exact number of location which each program will occupy and in consequence can make use of these numbers in the supervisor and translator programs. 
The proposed center of the first sentence is "system programs". The center remains the same in the second, third and forth sentences. Syntactic constraints are sufficient to establish the antecedent of "they" in the third sentence as "system programs". In the forth sentence, syntactic constraints only, however, are insufficient. Semantic constraints help here in assigning "system programs" as antecedent to "they". In the fifth sentence neither syntactic nor semantic constraints can resolve the ambiguity. The correct decision comes from proposing the center of the previous sentence, in this case "system programmers" (and not "programs"!), as the most likely antecedent.

\section{CONCLUSION}

The model proposed has two main advantages. First, it is an integrated model of different types of knowledge and uses existing techniques for anaphora resolution. Second, it incorporates a new approach for tracking the center, which proposes centers and subsequently antecedents with maximal likelihood. Since we regard our results still as preliminary, further research is necessary to confirm/improve the approach/model presented.

\section{ACKNOWLEDGEMENT}

I would like to express my gratitude to Prof. Pieter Seuren for his useful comments and to the Machine Translation Unit, Universiti Sains Malaysia, Penang, where a considerable part of the described rescarch has been carried out.

\section{REFERENCES}

[Aone \& McKee 93] Ch. Aonc, D. McKee language-independentanaphora resolution system for understanding multilingual texts. Proceedings of the 31st Annual Meeting of the ACL, The Ohio State University, Columbus, Ohio, 1993

[Allen87] J. Allen - Natural language understanding. The Benjamin/Cummings Publishing Company Inc., 1987

[Brennan et al. 87] S. Brennan, M. Fridman, C.
Pollard - A centering approach to pronouns. Proceedings of the 25th Annual Meeting of the ACL, Stanford, CA, 1987

[Carbonell \& Brown 88] J. Carbonell, R. Brown - Anaphora resolution: a multi-strategy approach. Proceedings of the 12 . International Conference on Computational Linguistics COLING'88, Budapest, 1988

[Dahl \& Ball 90] D. Dahl, C. Ball - Reference resolution in PUNDIT. Research Report CAITSLS-9004, March 1990. Center for Advanced Information Technology, Paoli, PA 9301

[Frederking \& Gehrke 87] R. Frederking, M. Gchrke - Resolving anaphoric references in a DRT-based dialogue system: Part 2: locus and Taxonomic inference. Sicmens AG, WISBER, Bericht Nr.17, 1987

[Grosz \& Sidner 86] B. Grosz, C. Sidner Attention, Intention and the structure of Discourse. Computational Linguistics, Vol. 12, 1986

[Hayes 81] P.J. Hayes - Anaphora for limited domain systems. Proceedings of the 7th IJCAI, Vancouver, Canada, 1981

[Hirst 81] G. Hirst - Anaphora in natural language understanding. Bcrlin Springer Verlag, 1981

[Hobbs 78] J. IIobbs - Resolving pronoun references. Lingua, Vol. 44, 1978

[Ingria \& Stallard 89] R. Ingria, D. Stallard - A computational mechanism for pronominal reference. Proceedings of the 27th Annual Mecting of the ACL, Vancouver, British Columbia, 1989

[Mitkov 93] R. Mitkov - A knowledge-based and sublanguage-oriented approach for anaphora resolution. Procecdings of the Pacific Asia Conference on Formal and Computational Linguistics, Taipei, 1993

[Preuß et al. 94] Preuß S., Schmitz B., Hauenschild C., Umbach C. - Anaphora Resolution in Machine Translation. In W. Ramm, P. Schmidt, J. Schiit火 (eds.) Studies in Machine Translation and Natural Ianguage Processing, Volume on "Discourse in Machine Translation"

[Rich \& LuperFoy 88] L. Rich, S. J uperFoy An architecture for anaphora resolution. Proccedings of the Second Conference on Applied Natural Language Processing, Austin, Texas, 1988

[Robert 89] M. Robert - Résolution de formes pronominales dans l'interface d'interogation d'une base de données. Thèse de doctorat. Faculté des sciences de Luminy, 1989

[Sidner 81] C.L. Sidner - Focusing for Interpretation of Pronouns. American Journal of Computational Linguistics, 7, 1981

[Walker 89] M. Walker - Evaluating discourse processing algorithms. Proceedings of the 27th Annual Mecting of the ACL, Vancouver, Columbia, 1989 